\title{
Selectivity in electron emission induced by ultrashort circularly polarized laser pulses
}

\author{
S. Walker $\odot$, L. Kolanz, J. Venzke $\odot$, and A. Becker \\ JILA and Department of Physics, University of Colorado, Boulder, Colorado 80309-0440, USA
}

(Received 28 July 2021; accepted 29 September 2021; published 19 October 2021)

\begin{abstract}
We theoretically investigate the ionization of neonlike atoms by an intense ultrashort circularly polarized laser pulse as a function of wavelength, spanning the regime from single-photon ionization to multiphoton and tunneling ionization. In order to determine the dependence of the ionization probabilities on the magnetic quantum number of the initial state, we perform $a b$ initio numerical calculations of the corresponding time-dependent Schrödinger equation in the single-active-electron approximation. Specific emphasis of our analysis is given to the intermediate-wavelength regime of about $50 \mathrm{~nm}$ to $300 \mathrm{~nm}$, in which the trend in the ionization probability of counterrotating electrons strongly differs from those for electrons in the other two initial magnetic sublevels. Theoretical analysis and numerical results indicate that the enhanced emission of counterrotating electrons occurs via photon absorption channels, which are only accessible for electrons rotating opposite to the rotation direction of the external electric field. Physical mechanisms behind the enhancement are identified as threshold effects, in which the emission into continuum states with low angular momentum quantum numbers is favored, and resonant enhanced ionization involving transitions via specific excited states in the atom. Numerical results showing the strong population in the relevant excited states, specifically those with low angular momentum quantum number, for the ionization of counterrotating electrons, and the overall similar trends in ionization and excitation for electrons with the other two initial magnetic quantum numbers support our interpretation. Overall, these effects lead to a change in the ionization ratio of co- to counterrotating electrons by two orders of magnitude from 10:1 to 1:10. This strong selectivity in the emission of electrons may lead to new opportunities in the generation of ultrashort spin-polarized electron pulses.
\end{abstract}

DOI: 10.1103/PhysRevResearch.3.043051

\section{INTRODUCTION}

Spin polarization of atomic photoelectrons is an important aspect in exploring the structure of atoms, molecules, and solids [1]. The generation of ultrashort spin-polarized electron pulses can be achieved by ionizing a gaseous atomic target with ultrashort circularly polarized laser pulses. These electron pulses are useful tools to characterize and probe chiral systems and magnetic properties of materials on an ultrafast timescale. Toward an understanding of the mechanisms behind the generation of spin-polarized electrons it is essential to study the sensitivity of the ionization probability to the sense of the electron's rotation in the initial state of the atom with respect to the helicity of the applied circularly polarized laser field $[2,3]$. Thus, it is interesting to identify laser parameter regimes in which the ionization rate strongly depends on the number and/or the sign of the magnetic quantum number in the initial state.

Spin polarization of photoelectrons and the related dependence of atomic photoionization on the relative rotation between electrons in the initial state and the applied field

Published by the American Physical Society under the terms of the Creative Commons Attribution 4.0 International license. Further distribution of this work must maintain attribution to the author(s) and the published article's title, journal citation, and DOI. has first been studied for single-photon ionization. While it was first believed that spin polarization of photoelectrons is a relativistic effect [4], Fano showed that electrons can be spin polarized in the nonrelativistic energy region as well [5]. In general, for single-photon ionization the emission of electrons corotating with respect to the rotation of the electric field of the applied field is more likely than that of counterrotating electrons. Stronger spin polarization was predicted for energy regions near Cooper minima. Experimental [6,7] and further theoretical [8] investigations supported these predictions by Fano. Preference of the emission of corotating over counterrotating electrons has also been found in the ionization of atoms prepared in Rydberg states with microwave radiation $[9,10]$. In the 1970s and 1980s the quest for sources generating spin-polarized electrons continued along with the development of laser technology. Significant electron spin polarization has been predicted and observed in two- and three-photon ionization of atomic targets [11-22]. At the corresponding laser frequencies the mechanism for spin polarization is related to intermediate resonances. The cross sections for (near-)resonant two- or three-photon ionization can be large if high-power lasers with narrow spectral bandwidth are available.

Using ultrashort laser pulses, fine-tuning of the laser frequency to specific resonances is generally not possible and (near-)resonant effects, previously observed with lasers having a long pulse duration, are often less pronounced in the ultrashort pulse regime. However, in the highly 
nonperturbative tunneling regime of strong-field ionization a different selectivity in the emission of electrons on the sense of the electron rotation in the initial state has been predicted recently [2]. In contrast to the general observations in the single-photon ionization regime, strong-field tunnel ionization yields larger ionization probabilities for counterrotating electrons than for those corotating with respect to the helicity of the applied field. The effect is due to nonadiabatic tunneling in which the two kinds of electrons have, contrary to the standard assumption in strong-field tunneling theory, nonzero velocities at the exit of the tunnel barrier. This prediction sparked significant experimental [3,23-30] and theoretical [28-71] interest over the last decade, including the observation of spin polarization of electrons of about $30 \%-50 \%$ for the interaction of atoms with laser pulses at $800 \mathrm{~nm}[3,27,34]$ and an even larger one at about $400 \mathrm{~nm}$ [29].

The role of excited states on the selectivity in the emission of counter- versus corotating electrons has been investigated in a few studies $[29,45,60,62,63,65,69,70]$. It has been shown that resonant excitation can lead to peaks in the helicity-dependent enhancement of ionization at wavelengths in the deep multiphoton regime [45]. On- and off-resonance two-photon ionization of an inner $p$-subshell electron has been discussed in the context of fluorescence polarization [63]. More recently, experimental and theoretical work has demonstrated that at wavelengths of about $400 \mathrm{~nm}$ Freeman resonances [72] provide a scheme to separate counterrotating electrons from corotating electrons in the photoelectron energy spectrum of xenon atoms [29]. In this case resonant ionization via an intermediate state close to the threshold, which is accessible to the counterrotating electrons only, leads to a selection in the electron emission at a specific kinetic energy. Therefore, the scheme provides an opportunity to generate spin-polarized electrons by energy gating. Furthermore, strong asymmetries in the ionization with left- and righthanded circularly polarized light for the lithium atom prepared in the polarized $2 p(m=+1)$ state have been observed [69]. In that combined experimental-theoretical study the emission of counterrotating electrons is found to be favored when the wavelength is tuned to the $2 p-3 s$ transition. In our recent theoretical study [70] we have more generally analyzed the impact of resonant transitions via excited states, that-due to the selection rules - can only be accessed by counterrotating electrons. These states act as doorway states in the few-photon ionization regime and are responsible for a dependence of the total ionization probability on the magnetic quantum number of the initial state which is stronger than those in both the single-photon and the tunneling regimes. The maximum ratios of total ionization of counter- over corotating electrons, found in the numerical calculations, were about ten to one, indicating the possibility to achieve a large selectivity in the overall electron emission.

In the previous short report [70] we focused on the surprisingly large change of the ionization ratio of counter- versus corotating electrons in the few-photon ionization regime. Here, we provide a more comprehensive discussion of the results over the broad wavelength regime from single-photon to multiphoton and tunneling ionization of neonlike atoms by ultrashort circularly polarized laser pulses. We identify the role of channels with different orbital angular momentum in the continuum near the ionization threshold and the importance of resonant transitions in different wavelengths regimes, also connecting the results and interpretation to earlier work. Furthermore, we compare the ionization probabilities for co- and counterrotating electrons to those of electrons from the initial state with magnetic quantum number $m=0$, which has been analyzed parenthetically in previous work only. Comparison of the results for excitation and ionization from all three initial magnetic levels supports the interpretation of the relevance of the additional pathways and the doorway states, accessible to the counterrotating electrons, in the intermediate-wavelength regime.

The remainder of the article is organized as follows: In Sec. II we outline the numerical methods used to solve the time-dependent Schrödinger equation for the interaction of neonlike atoms with an intense ultrashort circularly polarized pulse in three spatial dimensions. Next, in Sec. III A we first discuss the options to achieve a selectivity in electron emission via additional photon absorption channels, which are available for counterrotating electrons only. We then identify a few general trends in the ionization probabilities for electrons from the three different initial magnetic quantum levels. Comparison of the numerical results with those from lowest order perturbation theory and nonadiabatic tunneling theory let us identify the wavelength regions in which the additional pathways have a strong impact. Further analysis of the data then shows the importance of threshold effects (Sec. III B) and the relevance of resonant transitions via doorway states (Sec. III C). The article ends with a brief summary.

\section{NUMERICAL METHODS}

To study the dependence of strong-field ionization on the value and the sign of the magnetic quantum number, we have solved the time-dependent Schrödinger equation (TDSE) for the interaction of an atom with an intense laser pulse (we use Hartree atomic units $e=m_{e}=\hbar=1$ if not mentioned otherwise):

$$
i \frac{\partial}{\partial t} \Psi(\mathbf{r}, t)=\left[-\frac{\nabla^{2}}{2}+\mathbf{E}(t) \cdot \mathbf{r}+V(r)\right] \Psi(\mathbf{r}, t) .
$$

Here, $V(r)$ is a single-active electron (SAE) model potential for an electron in the outermost shell of an atom. In the present work we have considered ionization from the $2 p$ shell in a neonlike potential given by

$$
V(r)=-\frac{1}{r}-\frac{Z_{c} e^{-c r}}{r}-a_{1} e^{-b_{1} r}-a_{2} e^{-b_{2} r},
$$

where the first term represents the bare Coulomb potential, $Z_{C}=9$ is the remainder of the charge in the short-range part of the potential with $c=0.887$, and $a_{1}=-9.9286, a_{2}=$ $-5.995, b_{1}=1.3746$, and $a_{2}=3.7963$ are the parameters for the two exponential terms representing the $n=1$ and $n=2$ shell in the neonlike atom. The potential has been constructed using a method for benchmark tests between TDSE and time-dependent density functional theory calculations [73]. The first-ionization potential of $I_{2 p}=0.8353$ a.u. therefore matches the corresponding density functional theory potential while the experimental value for neon is 0.7927 a.u. The difference between theoretical and experimental values for the 
ionization potential does not impact the interpretation of the effects discussed in the present work.

For the circularly polarized laser pulse we set the vector potential of the applied field as [74]

$$
\mathbf{A}(t)=A_{0} \sin ^{2}\left(\frac{\pi t}{\tau}\right)\left[\sin \left(\omega_{A} t\right) \hat{\mathbf{x}}-\epsilon \cos \left(\omega_{A} t\right) \hat{\mathbf{y}}\right],
$$

where $A_{0}=\sqrt{c^{2} I /\left(1+\epsilon^{2}\right) \omega_{A}^{2}}, \tau=\frac{2 \pi N}{\omega_{A}}, c$ is the speed of light, $I$ is the peak intensity, $N$ is the number of cycles in the pulse, and $\omega_{A}$ is the central frequency of the vector potential. This ensures that the electric field integrates to zero. $\omega_{A}$ is determined such that the spectral distribution of the $E$ field matches the given physical central frequency $\omega_{E}$ [75]. The electric field is obtained by

$$
E(t)=-\frac{1}{c} \frac{\partial}{\partial t} A(t)
$$

We have considered a broad wavelength regime from $10 \mathrm{~nm}$, which corresponds to single-photon ionization, to $1000 \mathrm{~nm}$, corresponding to tunneling ionization. At all wavelengths we have applied intense laser pulses with a duration of 10 cycles and a peak intensity of $5 \times 10^{14} \mathrm{~W} / \mathrm{cm}^{2}$. Without loss of generality we have chosen left-handed circularly polarized pulses (i.e., $\epsilon=-1$ ). In each calculation we have prepared the atomic system in one of the three magnetic sublevels of the $2 p$ shell. Equation (1) has been solved in three spatial dimensions by using previously developed grid-based [76] and numerical basis-state methods [77,78]. At the end of each simulation we have determined the population in the excited states, characterized by the quantum numbers $n, l$, and $m$, via projection on the corresponding bound states of the atomic potential, which have been determined numerically. The ionization probability has then been evaluated as the difference between unity and the total probability left in the bound states.

In the grid-based method we have expanded $\Psi(\mathbf{r}, t)$ in spherical harmonics up to $l_{\max }=\left|m_{\max }\right|=40$ and discretized the radius using fourth-order finite difference. The wave function has been propagated in time with a time step of 0.05 a.u., on a grid with spacing of 0.05 a.u., maximum radius of 500 a.u., and exterior complex scaling on the outer 25 a.u. of the grid using the Crank-Nicolson method to propagate the wave function in time. This method was mainly used for the simulations at long wavelengths.

In the numerical basis state method we have also expanded $\Psi(\mathbf{r}, t)$ in spherical harmonics with $l_{\max }=\left|m_{\max }\right|=40$ in a box with maximum radius of 500 a.u. The basis was generated by diagonalizing the field-free Hamiltonian including a double-exponential complex absorbing potential on the outer 50 a.u. of the domain using a second-order finite-difference method with a grid spacing of 0.03 a.u. In the calculations we have chosen a maximum principal quantum number $n_{\max }=$ 500 corresponding to a maximum energy per angular momentum block of about 4.9 a.u. The wave function is propagated in time using the Crank-Nicolson method with a time step of 0.05 a.u. We account for outgoing boundary conditions and the results have been checked against those of the grid-based approach. Transitions to occupied states other than the initial state have been prohibited in the basis code calculations.
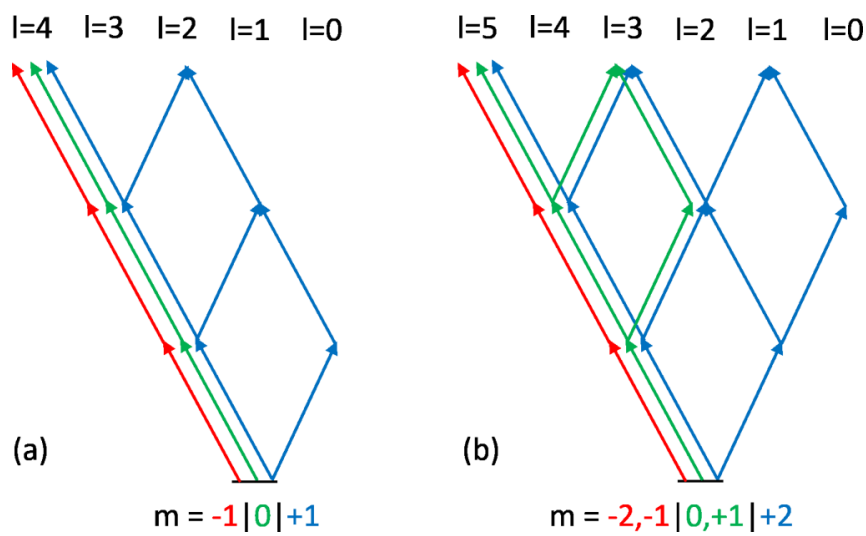

FIG. 1. Photon absorption channels for interaction of a lefthanded circularly polarized laser field $(\Delta l= \pm 1, \Delta m=-1)$ with an atom in initial (a) $p$ (red: $m=-1$, green: $m=0$, blue: $m=1$ ) and (b) $d$ state (red: $m=-2,-1$, green: $m=0,1$, blue: $m=2$ ).

\section{RESULTS AND DISCUSSION}

\section{A. Wavelength dependence: Overview}

\section{Selectivity in electron emission}

We first discuss concepts to enhance the emission probability of electrons from initial atomic states with different magnetic quantum numbers via photon absorption. The absorption of a photon from a left-handed circularly polarized field leads to changes of orbital angular and magnetic quantum numbers of $\Delta l= \pm 1$ and $\Delta m=-1$. The schematic representations in Fig. 1 show the allowed pathways for the absorption of the first three photons from initial (a) $p$ and (b) $d$ states.

In the case of an initial $p$ state [Fig. 1(a)] for electrons in each of the three magnetic sublevels transitions with $\Delta l=1$ are allowed leading to a successive increase of the orbital angular quantum number with the absorption of each photon. A selectivity along this pathway is therefore only possible either due to differences in the transition amplitudes or via intermediate resonant states. The former one is the cause for a strong suppression of electron emission from the initial $m=0$ quantum level in the few- and multiphoton ionization regime with circularly polarized laser light (see Sec. III A 2). If energies of excited states in the atom depend on the total angular quantum number, then the ionization probability from a specific initial $m$ level can be enhanced by fine-tuning the laser frequency to an intermediate resonance [11]. Since differences between these energy levels are usually small such selection requires a small laser bandwidth and an enhancement is only effective for long laser pulse durations. For the interaction with ultrashort laser pulses such effects related to fine-tuning of the laser frequency are therefore usually rather small.

Figure 1(a) however also shows that there are additional photon absorption channels for the counterrotating electrons (here, electrons in the initial $m=1$ level; blue arrows). For example, for a counterrotating electron the absorption of a (left-handed circularly polarized) photon induces a change in magnetic quantum number to $m=0$ and therefore transitions into both $l=2$ and $l=0$ levels are allowed. In contrast, for the other two initial sublevels only the transition to $l=2$ is possible. Similar considerations hold for the absorption 
of additional photons. If the probability via these additional channels can be enhanced, we should therefore expect a strong selectivity of the emission of counterrotating electrons in the interaction with a circularly polarized laser field. As our results below indicate this can be achieved either near the thresholds of photon absorption (Sec. III B) or via intermediate resonances which act as doorway states accessible for the counterrotating electrons only (Sec. III C). These additional pathways to ionization have been also mentioned in Refs. [29,60,62,63,65,69,70]. Resonant transitions via different pathways for counterrotating electrons in two-photon ionization have been discussed in Refs. [60,63]. A Freeman resonance close to the threshold [72] has been used in the selection of counterrotating electrons via energy gating by $\mathrm{Xu}$ et al. [29]. A distinct maximum for the ionization probability of counterrotating electrons has been observed at wavelengths near the $2 p-3 s$ transition for lithium atoms prepared in the polarized $2 p(m=+1)$ state recently [69]. More generally, an enhancement of the additional pathways in the few-photon ionization regime as the cause for the reversal in emission of counter- over corotating electrons has been identified in our previous work [70]. Since selective emission via these additional pathways does not rely on a splitting of energy levels, the enhancement can be achieved independently of the duration of the applied laser pulse, which is interesting for a generation of ultrashort spin-polarized electron pulses.

With our numerical results presented below we study the applicability of these concepts for the initial $2 p$ state in the neon atom. Reasoning along similar lines as above can be however done for initial states with a larger orbital angular momentum number as well, as is illustrated for an initial $d$ state in Fig. 1(b). Besides the $\Delta l=1$ pathway, which is open for electrons from each of the five initial $m$ levels, additional pathways are available for electrons from the initial levels with $m=0,1$ (red arrows) and even more for those from the $m=2$ state (blue arrows). Thus, by identifying specific frequency regions in which the transition probability via one or some of these additional pathways is large one can expect an enhancement in the emission of certain groups of electrons.

\section{General trends}

In Fig. 2 we present the numerical results for the ionization probability from the three $2 p$ orbitals of a neonlike atom as a function of wavelength in a 10-cycle left-handed circularly polarized laser pulse at peak intensity of $5 \times 10^{14} \mathrm{~W} / \mathrm{cm}^{2} . P_{+}$, where the index denotes the magnetic quantum number of the initial orbital ( $m=1,2 p_{+}$; stars with line), corresponds to the ionization probability for counterrotating electrons, while $P_{-}$ (circles with line) is the one for corotating electrons and $P_{0}$ (squares with line) denotes the ionization probability for the initial state with $m=0$.

There are a few general trends which we will discuss before we further analyze more specific features in the next subsections. In the short-wavelength regime the single-photon ionization probability for each of the three subshells increases approximately proportional to $\lambda^{7 / 2}$, as is expected at large photon energies based on the Born approximation [79]. In agreement with earlier work [5,11] for single-photon transitions corotating electrons are easier to ionize than

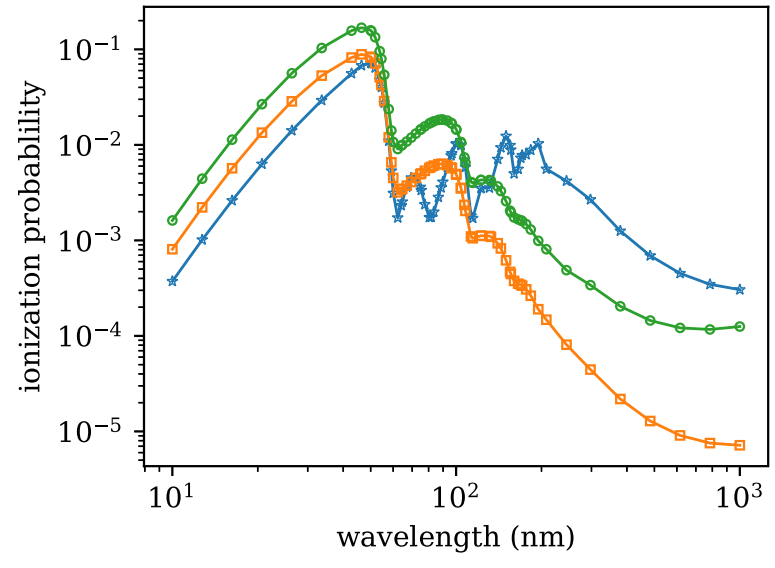

FIG. 2. Ionization probabilities $P_{+}$(stars with line), $P_{0}$ (squares with line), and $P_{-}$(circles with line) as function of wavelength for neonlike atom at $5 \times 10^{14} \mathrm{~W} / \mathrm{cm}^{2}$ interacting with 10 -cycle lefthanded circularly polarized laser pulses.

counterrotating electrons, while the ionization probability for the initial orbital with $m=0$ remains in between those for the other two subshells. The differences in the trends of the ionization probabilities near the one-photon threshold will be discussed in Sec. III B.

In the two-, few-, and multiphoton ionization regime the dependencies of $P_{-}$and $P_{0}$ on the wavelength remain rather similar. As discussed in Sec. III A 1 [cf. Fig. 1(a)], for electrons in initial $p_{-}$and $p_{0}$ states absorption of photons is allowed through the pathway with $\Delta l=1$ only. Therefore, the absorption of $N$ photons results in the final state with $l_{f}=N+l$ and $m_{f}=m-N$, where $l$ is the angular momentum quantum number of the initial state. In lowest order perturbation theory the transition amplitudes for both initial states share identical intermediate reduced matrix elements and differ from each other only by a $3 j$ symbol. A perturbative scaling law for the ratio of ionization probabilities via the $\Delta l=+1$ pathway for electrons in initial states $(l, m)$ and $\left(l, m^{\prime}\right)$ is therefore given by

$$
\frac{P_{m}}{P_{m^{\prime}}}=\prod_{k=1}^{N_{\min }} \frac{\left(\begin{array}{ccc}
l+k & 1 & l+k-1 \\
k-m & -1 & m+1-k
\end{array}\right)^{2}}{\left(\begin{array}{ccc}
l+k & 1 & l+k-1 \\
k-m^{\prime} & -1 & m^{\prime}+1-k
\end{array}\right)^{2}},
$$

where $N_{\min }=\left\lceil I_{p} / \omega\right\rceil$ is the minimum number of photons absorbed and $I_{p}$ is the field-free ionization potential. Using

$$
\begin{aligned}
& \left(\begin{array}{ccc}
l+k & 1 & l+k-1 \\
k-m & -1 & m+1-k
\end{array}\right) \\
& \quad=(-1)^{l-m} \sqrt{\frac{(2 k+l-m-1)(2 k+l-m)}{(2 k+2 l-1)(2 k+2 l)(2 k+2 l+1)}},
\end{aligned}
$$

we get

$$
\begin{aligned}
\frac{P_{m}}{P_{m^{\prime}}} & =\prod_{k=1}^{N_{\min }} \frac{(2 k+l-m-1)(2 k+l-m)}{\left(2 k+l-m^{\prime}-1\right)\left(2 k+l-m^{\prime}\right)} \\
& =\frac{\left(l-m^{\prime}\right) !\left(2 N_{\min }+l-m\right) !}{(l-m) !\left(2 N_{\min }+l-m^{\prime}\right) !} .
\end{aligned}
$$



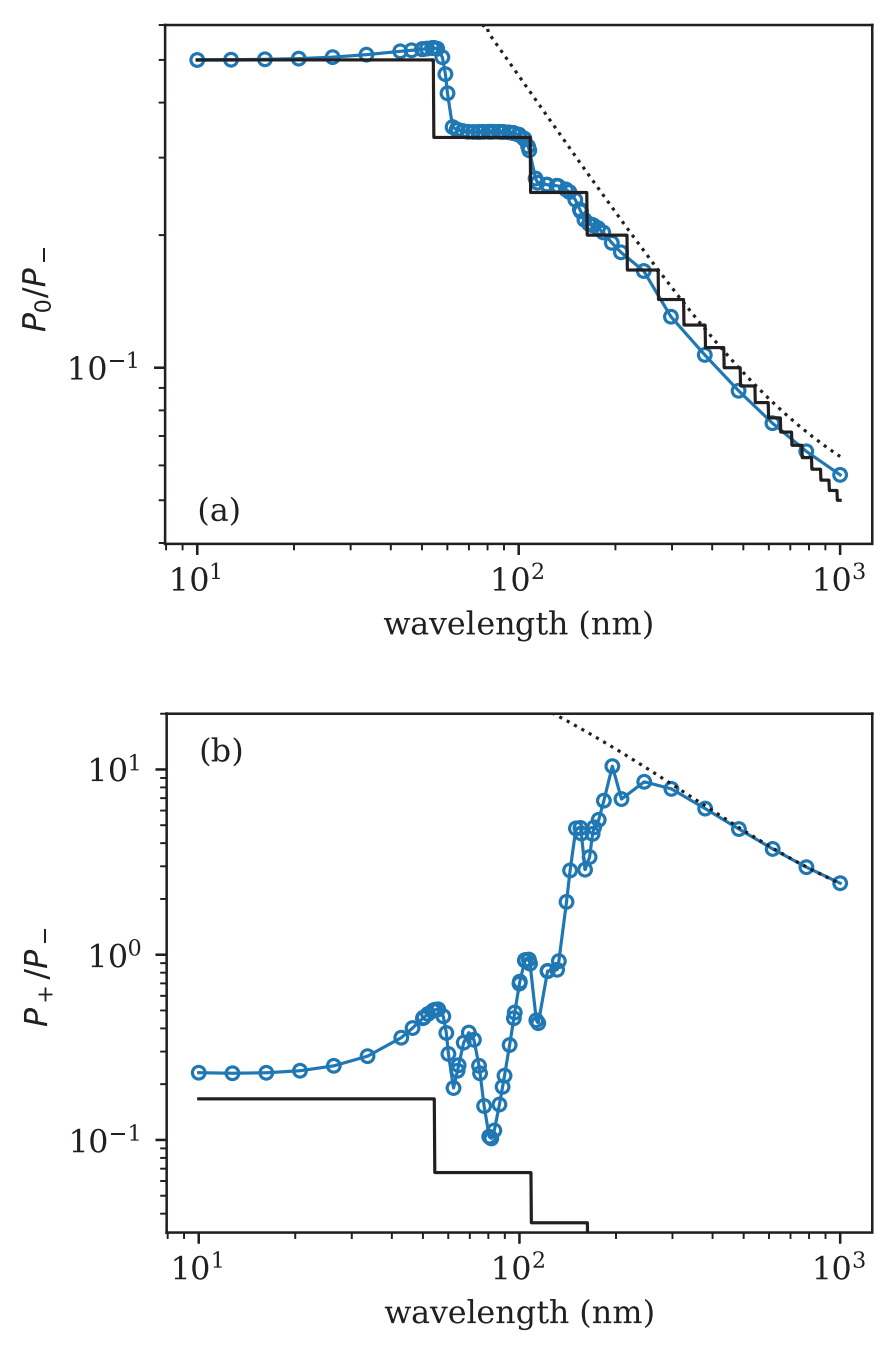

FIG. 3. Comparison of numerical results (circles with line) for the ratios (a) $P_{0} / P_{-}$and (b) $P_{+}\left(l_{f}=N_{\min }+1\right) / P_{-}$with the scaling laws from lowest order perturbation theory for $\Delta l=1$ pathway [solid lines; Eqs. (8) and (9)] and nonadiabatic tunneling predictions [dotted lines; Eqs. (10) and (12)] as function of wavelength. Laser parameters are the same as in Fig. 2.

Thus, for an initial $p$ state with $l=1$ we have

$$
\frac{P_{0}}{P_{-}}=\frac{1}{N_{\min }+1}
$$

and

$$
\frac{P_{+}\left(l_{f}=N_{\min }+1\right)}{P_{-}}=\frac{1}{\left(N_{\min }+1\right)\left(2 N_{\min }+1\right)},
$$

where $P_{+}\left(l_{f}=N_{\min }+1\right)$ is the perturbative ionization probability for counterrotating electrons along the $\Delta l=+1$ pathway only.

The comparison in Fig. 3(a) shows that the numerical results for $P_{0} / P_{-}$(circles with line) overall agree well with the perturbative scaling law [solid line; Eq. (8)] at the shorter wavelengths. The differences near the photon absorption thresholds are due to the bandwidth of the short pulse used in the calculations. The deviation at large wavelengths indicates the transition to the tunneling regime. In this regime the numerical results for $P_{0} / P_{-}$agree rather well with predictions based on nonadiabatic tunneling theory [dotted line in Fig. 3(a)] [2,31],

$$
\begin{aligned}
\frac{P_{0}}{P_{-}}= & \sqrt{\frac{I}{16 I_{p}^{3}} \frac{1+\gamma^{2}}{\zeta_{0}^{2}+\gamma^{2}}} \\
& \times \frac{\gamma^{3}\left(\zeta_{0}-1\right)^{2}\left(\zeta_{0}+1\right)}{\left(\sqrt{\zeta_{0}^{2}+\gamma^{2}}-\zeta_{0} \sqrt{1+\gamma^{2}}\right)^{2}},
\end{aligned}
$$

where $I$ is the laser intensity, $\gamma$ is the Keldysh parameter, and $\zeta_{0}$ is determined by

$$
\sqrt{\frac{\zeta_{0}^{2}+\gamma^{2}}{1+\gamma^{2}}}=\tanh \left(\frac{1}{1-\zeta_{0}} \sqrt{\frac{\zeta_{0}^{2}+\gamma^{2}}{1+\gamma^{2}}}\right) .
$$

In contrast, the results for $P_{+} / P_{-}$(circles with line) in Fig. 3(b) show a deviation from both the perturbative scaling law [solid line; Eq. (9)] and the prediction of the nonadiabatic tunneling theory (dotted line) [2,31],

$$
\frac{P_{+}}{P_{-}}=\left(\frac{\sqrt{\zeta_{0}^{2}+\gamma^{2}}+\zeta_{0} \sqrt{1+\gamma^{2}}}{\sqrt{\zeta_{0}^{2}+\gamma^{2}}-\zeta_{0} \sqrt{1+\gamma^{2}}}\right)^{2}
$$

in the intermediate two- and few-photon ionization regime. Here, $P_{+} / P_{-}$changes by two orders of magnitude, reversing from 1:10 to 10:1. An enhancement of the emission of counterrotating electrons in the few-photon ionization regime has also been observed in numerical results for strong-field detachment of $\mathrm{F}^{-}$[62]. As we will discuss in the next subsections, for the present results the strong variation in the ratio can be explained as due to the impact of the additional pathways available for the counterrotating electrons [cf. Fig. 1(a)]. From the results in Fig. 3(b) we can identify that these pathways should have a strong impact at the one- and two-photon ionization thresholds, the region at about $70 \mathrm{~nm}$, and the few-photon ionization regime.

Before proceeding, we note that the present numerical results agree well with the predictions of the nonadiabatic tunneling theory at long wavelengths. A similar degree of agreement has been reported before for results from an analytical $R$-matrix theory [35], 2D-TDSE calculations [37], and the $R$-matrix with time dependence method [62]. Furthermore, the results of the analytical $R$-matrix theory [35] indicate a maximum in the $P_{+} / P_{-}$ratio as well, which occurs in a wavelength regime close to that one where the maximum is found in the present results. Finally, we note that the trend of the results for $P_{+}$and $P_{-}$in Figs. 2 and 3(b) near $1000 \mathrm{~nm}$ may indicate that the relative emission probability of counter- versus corotating electrons reverses at even longer wavelengths. Such a reversal has been predicted in past theoretical studies $[45,64]$.

\section{B. Ionization thresholds and two-photon ionization}

At wavelengths near the one-photon ionization threshold the relative probability for emission of counterrotating electrons increases as compared to those from the initial levels with $m=-1$ and $m=0$. This can be seen from the ratios $P_{+} / P_{-}$[Fig. 4(b); stars with line] and $P_{+} / P_{0}$ [Fig. 4(c); stars with line]. The enhancement is due to the fact that for small photoelectron energies the transition in the continuum 

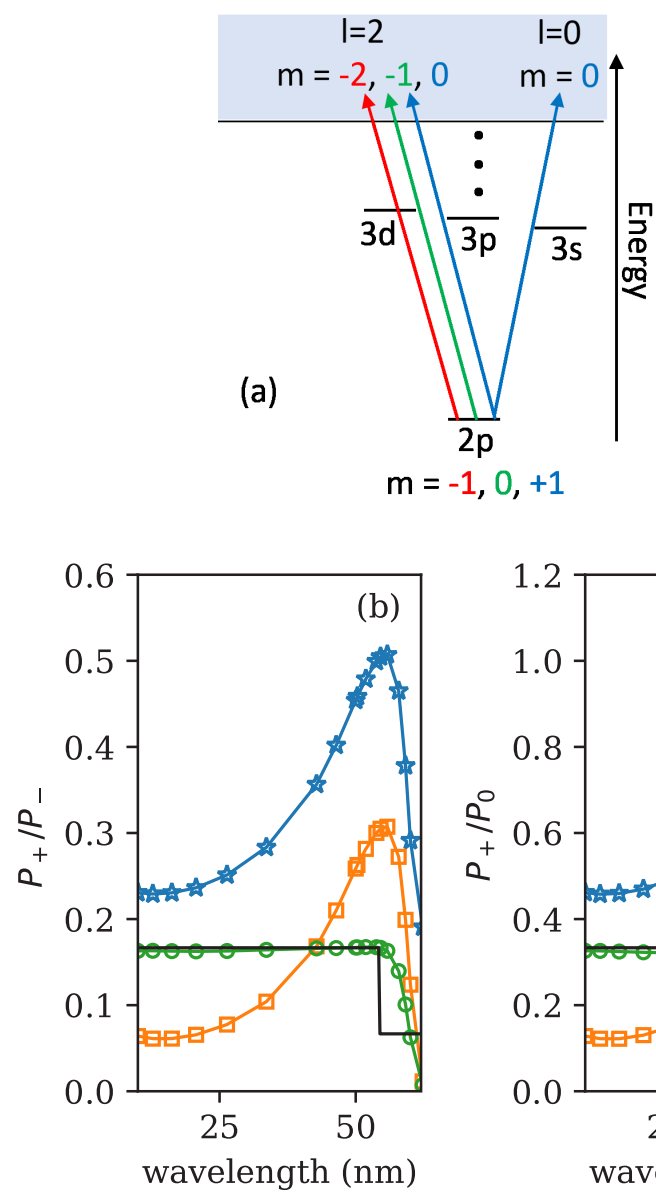
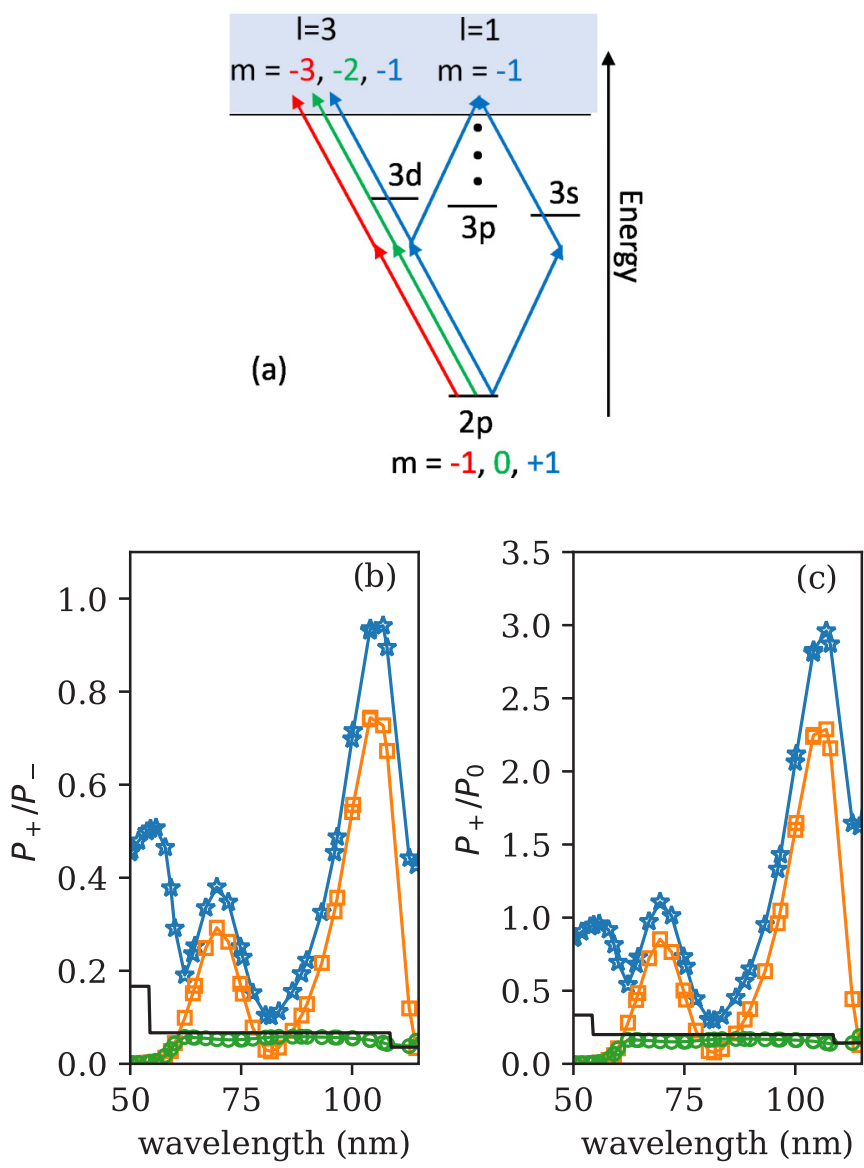

FIG. 5. Upper row: (a) Channels for two-photon ionization. Lower row: Comparison of ratios (b) $P_{+} / P_{-}$(stars with line), $P_{+}(l=$ $3) / P_{-}$(circles with line), and $P_{+}(l=1) / P_{-}$(squares with line), as well as (c) $P_{+} / P_{0}$ (stars with line), $P_{+}(l=3) / P_{0}$ (circles with line), and $P_{+}(l=1) / P_{0}$ (squares with line) as function of wavelength. Also shown are the predictions in lowest order perturbation for the $\Delta l=1$ channels (lines without symbols).

emission of counterrotating electrons into the final channel with the lowest possible angular momentum quantum number enhances the ratio of $P_{+} / P_{-}$and $P_{+} / P_{0}$ near the threshold [Figs. 5(b) and 5(c)].

The impact of the channels with lowest angular momentum quantum number in the final state can be also investigated via photoelectron angular distributions. For ionization along the $\Delta l=1$ pathway, available for all electrons, we expect an angular distribution proportional to the spherical harmonics $\left|Y_{2}^{m-1}\right|^{2}$ (for single-photon ionization) and $\left|Y_{3}^{m-2}\right|^{2}$ (for twophoton ionization). As can be seen, from the comparison of the numerical results in Fig. 6, obtained at the one-photon (left column; $53.8 \mathrm{~nm}$ ) and the two-photon threshold (right column; $104.12 \mathrm{~nm}$ ), this is the case for ionization from both $2 p_{0}$ [panels (b) and (e)] and $2 p_{-1}$ [panels (c) and (f)]. In contrast, the angular distributions for the counterrotating electrons at the one-photon threshold [Fig. 6(a)] show signatures of an interference. Specifically, in the projections it is seen that the node that would occur for a pure $\left|Y_{2}^{0}\right|^{2}$ distribution is not present. This indicates the presence of an interference with a spherical harmonic having a different, here lower, angular 

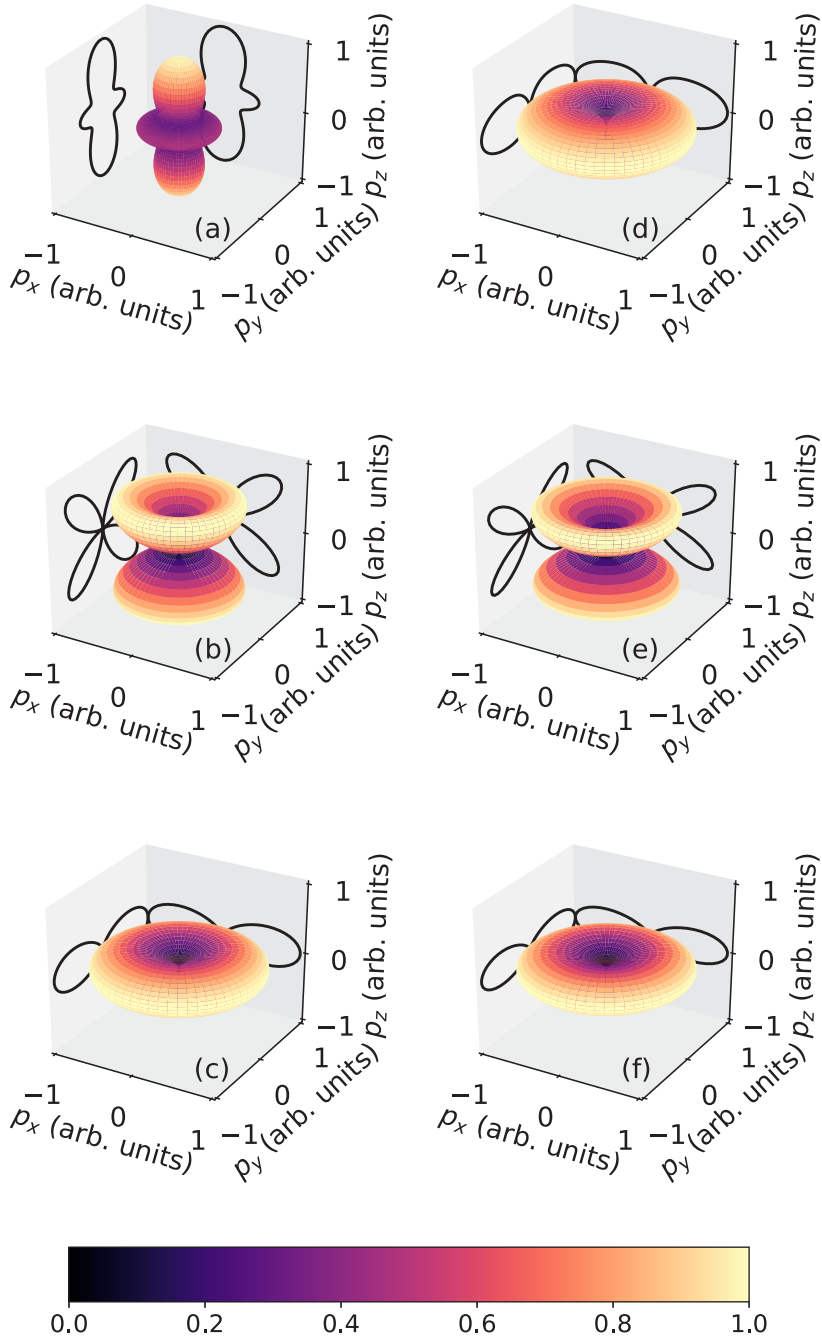

FIG. 6. Comparison of photoelectron angular distributions for ionization from initial state $m=1$ [(a), (d)], $m=0$ [(b), (e)], and $m=-1$ [(c), (f)] near one-photon [(a)-(c): $53.8 \mathrm{~nm}]$ and two-photon [(d)-(f): $104.12 \mathrm{~nm}$ ] threshold.

momentum, as expected. At the two-photon threshold the contribution from $l=3$ is significantly smaller than that from $l=1$ so interference is barely visible in the photoelectron angular distributions [Fig. 6(d)].

The enhancement in the $P_{+}$yield and the corresponding ratios at a wavelength of about $70 \mathrm{~nm}$ in the two-photon ionization regime is due to resonant ionization via the intermediate $3 s$ doorway state. While transition into the $3 d$ state is allowed from all three initial states via the absorption of one left-handed circularly polarized photon, the $3 s$ state is only accessible for electrons in the initial $2 p_{-1}$ state [cf. Fig. 1(a)]. Indications for the impact of the doorway state are given by the ratios $P_{+}\left(l_{f}=1\right) / P_{-}$and $P_{+}\left(l_{f}=1\right) / P_{0}$ in Figs. 5(b) and $5(\mathrm{c})$ and the large population in the $3 s$ state for ionization from the initial $2 p_{+}$state at the end of the pulse [Fig. 7(a)]. The relative importance of resonant transition via $s$ and $d$ states in the two-photon ionization regime has also been discussed for interaction of counterrotating electrons prepared in the excited $2 p$ state in the hydrogen atom [60]. Similarly, an enhancement

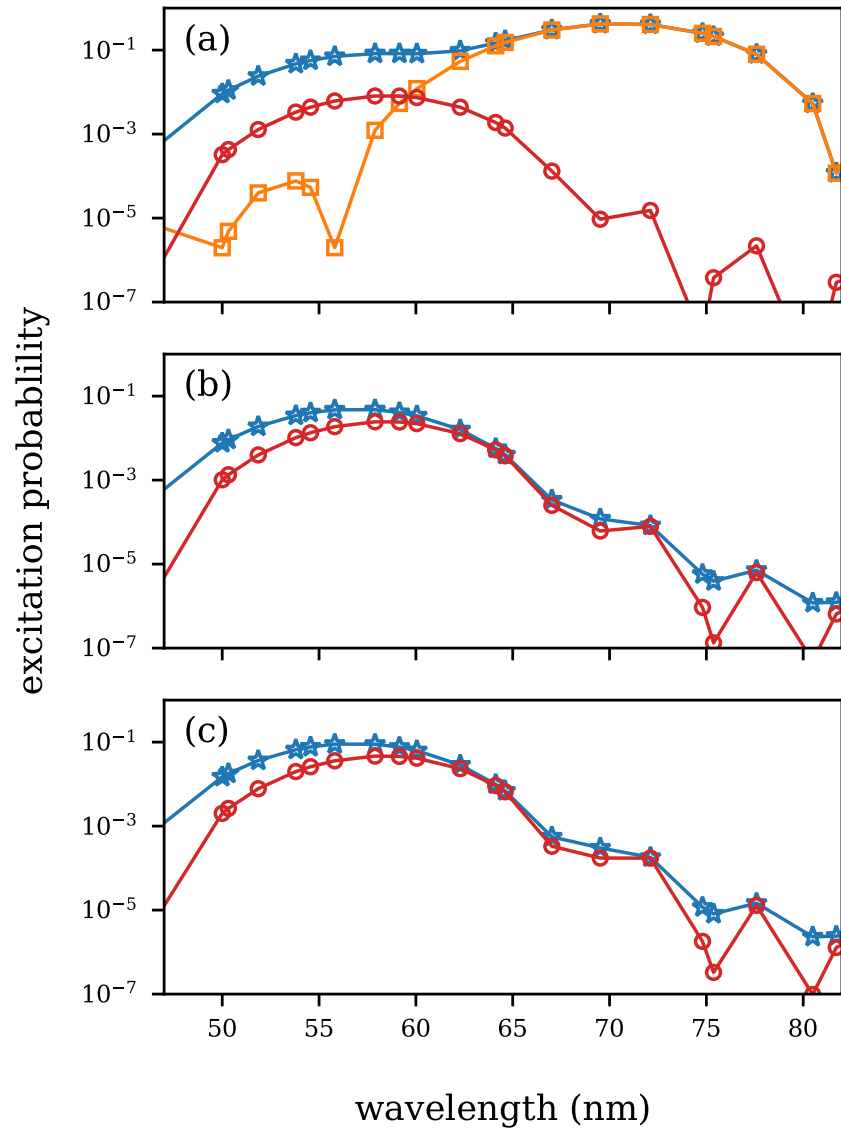

FIG. 7. Excitation probabilities in $3 s$ (orange squares), $3 d$ (red circles), and all states (blue stars) for ionization from initial states (a) $2 p_{1}$, (b) $2 p_{0}$, and (c) $2 p_{-1}$ for neonlike atom at $5 \times 10^{14} \mathrm{~W} / \mathrm{cm}^{2}$ interacting with 10 -cycle laser pulses.

in the emission of counterrotating electrons has been observed for a resonant $2 p-3 s$ transition in the three-photon ionization of lithium atoms, prepared in the polarized $2 p(m=+1)$ state [69]. Together, the data clearly support the interpretation that the resonant ionization pathway, which is accessible for the counterrotating electrons only, is the origin for the enhancement in the $P_{+}$yield. The comparison with the final populations in the states of the $n=3$ level for the other two initial states in Figs. 7(b) and 7(c) shows that in those cases only the $3 d$ state is populated and the population decreases as a function of wavelength in the two-photon ionization regime.

\section{Doorway states in few-photon ionization regime}

In contrast to the two-photon ionization regime, fewphoton ionization states in the $n=3$ level can only be accessed by the counterrotating electrons, as shown in the two schemes in Fig. 8. Corresponding to each of these additional pathways one can expect resonant enhanced ionization in the $P_{+}$yield. In contrast, there are no pathways for resonant enhanced ionization via the $n=3$ states for electrons in the other initial states.

The importance of the resonant transitions is supported by the results of our numerical calculations in Fig. 9. The comparison of the total and $3 l$ excitation probabilities for the three 

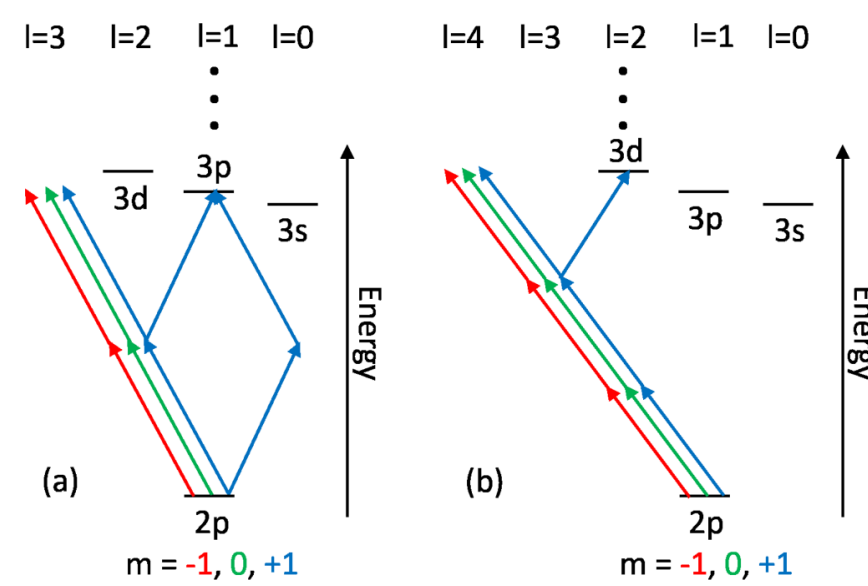

FIG. 8. (a) Two- and (b) three-photon transitions into doorway states in the $n=3$ shell from initial $2 p_{1}$ state. Other nonresonant transitions are also shown using same arrow styles as in Fig. 1.

initial states at the end of the pulse shows the stronger role of the overall excitation for counterrotating electrons [Fig. 9(a)] as compared to the other initial states [Figs. 9(b) and 9(c)] in the wavelength regime between $100 \mathrm{~nm}$ and $200 \mathrm{~nm}$.

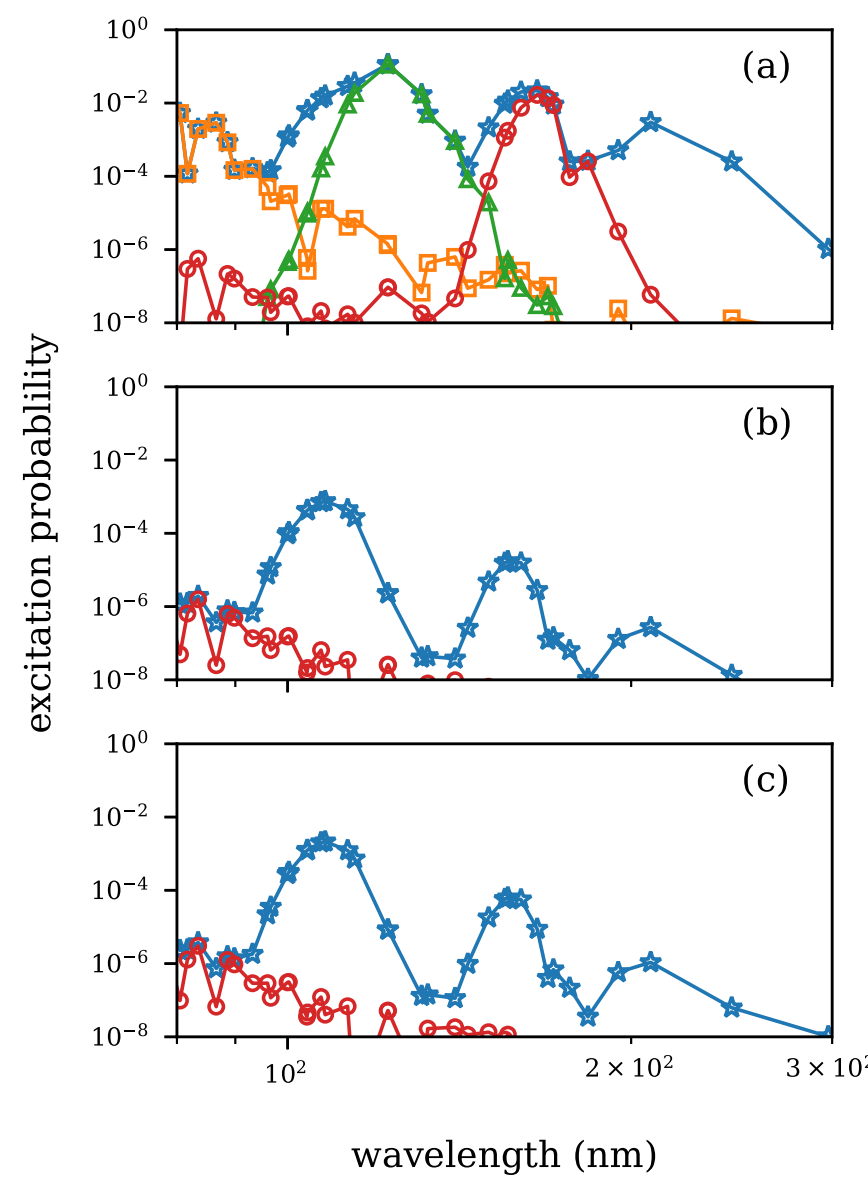

FIG. 9. Comparison of excitation probabilities in $3 s$ (orange squares), $3 p$ (green triangles), $3 d$ (red circles) with total excitation probability (blue stars) from initial states (a) $2 p_{1}$, (b) $2 p_{0}$, and (c) $2 p_{-1}$ for neonlike atom with 10 -cycle laser pulses at peak intensity $5 \times 10^{14} \mathrm{~W} / \mathrm{cm}^{2}$.

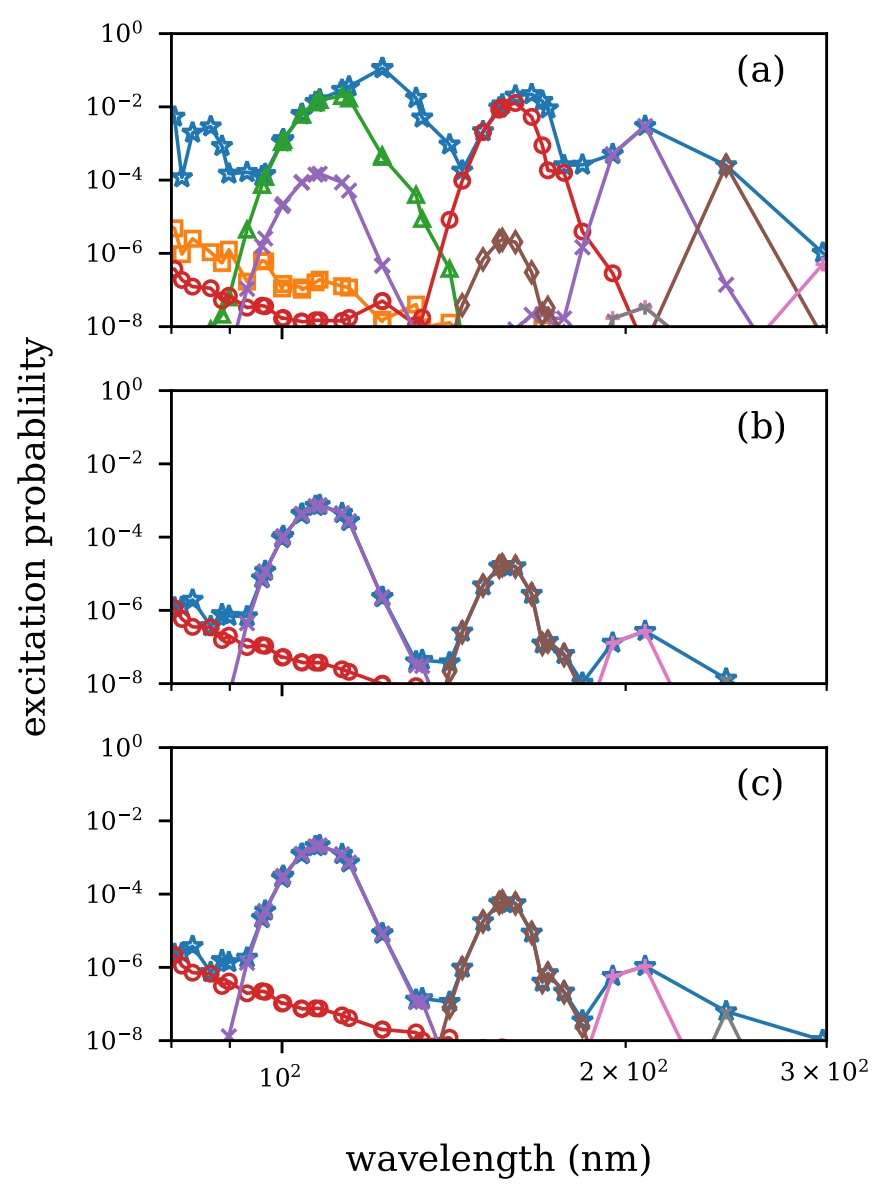

FIG. 10. Same as Fig. 9 but for $n s$ (orange squares), $n p$ (green triangles), $n d$ (red circles), $n f$ (purple crosses), $n g$ (brown diamonds), $n h$ (pink plus signs), and $n i$ (gray 3-pointed stars) states with $n \geqslant 4$. Total excitation probabilities are represented by blue 5 -pointed stars.

It is also seen that a major part of the total excitation arises from populations in either the $3 p$ level (two-photon resonant transition) or the $3 d$ level (three-photon resonant absorption). In contrast, there is only a very small final population in the $3 d$ state for the other two initial states. This population arises from one-photon transitions due to the broad bandwidth of the ultrashort pulse. Similarly, we see some small population in the $3 s$ state due to one-photon absorption for the initial $2 p_{1}$ state.

The remaining part of the total excitation probabilities for the counterrotating electrons as well as the contributions for the other initial states arise from higher excited states. This is shown in Fig. 10, where we present the corresponding populations in states with $n \geqslant 4$, separately for each $l$ quantum number up to $l=6$ ( $i$ states). The excitations for the initial states with (b) $m=0$ and (c) $m=-1$ both solely arise from the $f$ (at about $110 \mathrm{~nm}), g(\sim 170 \mathrm{~nm}), h(\sim 200 \mathrm{~nm}$ ), and $i$ levels $(\sim 240 \mathrm{~nm})$, confirming that the mechanisms and pathways to excitation and ionization from these two states are similar. The population of levels with successively larger $l$ values in separate regimes, as the wavelength increases, is in agreement with the interpretation of a resonant enhancement along the $\Delta l=1$ pathway. In contrast, the results for the counterrotating electrons [Fig. 10(a)] again indicate the strong 
impact of the additional pathways. In this case the populations in the excited states along the $\Delta l=1$ pathway do not provide significant contributions to the total excitation probability. For example, the population in the $n f$ states (purple crosses) at about $110 \mathrm{~nm}$ for initial state $m=1$ is smaller than the populations in the corresponding states for the interaction with the other two initial states. However, its contribution to the total excitation probability is negligible. Instead, the populations in the excited states with lower $l$ are, in general, by about two orders of magnitude stronger than those with the higher $l$ 's. This observation agrees with theoretical results for on-resonance two-photon ionization of an inner atomic $n p$ subshell by a circularly polarized light field [63].

\section{SUMMARY}

To summarize, we have performed $a b$ initio numerical calculations of the time-dependent Schrödinger equation to study the dependence of the interaction of neonlike atoms with an intense ultrashort circularly polarized laser pulse on the magnetic quantum number of the initial state. In our analysis we have considered a broad wavelength regime, ranging from single-photon to tunneling ionization. The numerical results for ionization of electrons corotating with respect to the rotation direction of the field and those from the initial level with $m=0$ agree well with the predictions from lowest order perturbation theory at short and nonadiabatic tunneling theory [2,31] at long wavelengths. In contrast, the data for the counterrotating electrons deviate significantly from both these predictions in the intermediate-wavelength regime.

At the one- and two-photon ionization thresholds the enhancement in the emission of counterrotating electrons can be explained via the transition into channels with low angular momentum quantum number in the continuum, which are accessible for these types of electrons only. Further increase in the ionization yield for counterrotating electrons (Fig. 2) and in the ionization ratio of counter- over corotating electrons [Fig. 3(b)] in the two- and few-photon ionization regime comes along with much stronger excitation probabilities found for the interaction of counterrotating electrons with a circularly polarized short laser pulse. Together with the finding that the population is mainly in excited states with lower angular momentum quantum number our results indicate that resonant enhanced ionization via specific pathways and doorway states, which are accessible for the counterrotating electrons only, is the physical mechanism behind the enhancement. The overall similar trends in ionization and excitation probabilities for initial states with $m=0$ and $m=1$, from which none of the doorway states can be excited, further support this interpretation.

The additional pathways for counterrotating electrons exist in all atoms with ground states having $l \geqslant 1$ and may therefore provide an alternative route toward the generation of ultrashort spin-polarized electron pulses. Energy gating of the photoelectrons, as proposed and demonstrated in [29], can even further enhance the selectivity in the ionization process. Increasing the wavelength beyond the few-photon ionization regime, the excitation probability becomes less relevant due to the large number of photons needed for resonant transitions into excited states close to the threshold. Correspondingly, the relative strength of the emission of counterrotating over corotating electrons gets significantly smaller, decreasing from a ratio of ten to one at $250 \mathrm{~nm}$ to less than three to one in the tunneling regime at $1000 \mathrm{~nm}$ [Fig. 3(b)].

\section{ACKNOWLEDGMENTS}

This work was primarily supported by a grant from the U.S. Department of Energy, Division of Chemical Sciences, Atomic, Molecular, and Optical Sciences Program (Award No. DE-SC0001771). We also acknowledge a grant from the U.S. National Science Foundation (Award No. 1734006) for computer resources used for part of the present calculations.
[1] J. Kessler, Polarized Electrons (Springer, Berlin, 1985).

[2] I. Barth and O. Smirnova, Nonadiabatic tunneling in circularly polarized laser fields: Physical picture and calculations, Phys. Rev. A 84, 063415 (2011).

[3] A. Hartung, F. Morales, M. Kunitski, K. Henrichs, A. Laucke, M. Richter, T. Jahnke, A. Kalinin, M. Schöffler, L. P. H. Schmidt, M. Ivanov, O. Smirnova, and R. Dörner, Electron spin polarization in strong-field ionization of xenon atoms, Nat. Photonics 10, 526 (2016).

[4] R. H. Pratt, A. Ron, and H. K. Tseng, Atomic photoelectric effect above $10 \mathrm{keV}$, Rev. Mod. Phys. 45, 273 (1973).

[5] U. Fano, Spin orientation of photoelectrons ejected by circularly polarized light, Phys. Rev. 178, 131 (1969).

[6] G. Baum, M. S. Lubell, and W. Raith, Spin-Orbit Perturbation in Heavy Alkali Atoms, Phys. Rev. Lett. 25, 267 (1970).

[7] U. Heinzmann, J. Kessler, and J. Lorenz, Wavelength Dependence of the Fano Effect, Phys. Rev. Lett. 25, 1325 (1970).

[8] N. A. Cherepkov, Angular distribution and spin orientation of photoelectrons ejected by circularly polarized light, Phys. Lett. A 40, 119 (1972).
[9] K. Rzazewski and B. Piraux, Circular Rydberg orbits in circularly polarized microwave radiation, Phys. Rev. A 47, R1612 (1993).

[10] J. Zakrzewski, D. Delande, J.-C. Gay, and K. Rzazewski, Ionization of highly excited hydrogen atoms by a circularly polarized microwave field, Phys. Rev. A 47, R2468 (1993).

[11] P. Lambropoulos, Spin-Orbit Coupling and Photoelectron Polarization in Multiphoton Ionization of Atoms, Phys. Rev. Lett. 30, 413 (1973).

[12] P. S. Farago and D. W. Walker, Two-photon ionization of trivalent atoms as a source of polarized electrons, J. Phys. B 6, L280 (1973).

[13] P. Lambropoulos, On producing totally polarized electrons through multiphoton ionization, J. Phys. B 7, L33 (1974).

[14] P. S. Farago, D. W. Walker, and J. S. Wykes, Remarks on photoelectron polarization in multiphoton ionization of alkali atoms, J. Phys. B 7, 59 (1974).

[15] P. Lambropoulos and M. R. Teague, Two-photon ionization with spin-orbit coupling, J. Phys. B 9, 587 (1976). 
[16] M. R. Teague and P. Lambropoulos, Three-photon ionization with spin-orbit coupling, J. Phys. B 9, 1251 (1976).

[17] E. H. A. Granneman, M. Klewer, K. J. Nygaard, and M. J. V. d. Wiel, Polarization effects in resonant two-photon ionization of caesium, J. Phys. B 9, L87 (1976).

[18] E. H. A. Granneman, M. Klewer, and M. J. V. d. Wiel, Twophoton ionization of caesium via the $6^{2} \mathrm{P}_{3 / 2}$ intermediate state: Analysis of photoelectron spin polarization and saturation effects, J. Phys. B 9, 2819 (1976).

[19] G. Baravian, J. Godart, and G. Sultan, Resonant multiphoton ionization of neon. Experimental determination of the polarizability of the $3 p^{\prime}\left[\frac{1}{2}\right]_{1}$ level, Phys. Rev. A 14, 761 (1976).

[20] A. I. Andrjushin and A. E. Kazakov, Polarised electrons and two-photon-resonant ionisation of unpolarised atoms, J. Phys. B 13, L609 (1980).

[21] S. N. Dixit, P. Lambropoulos, and P. Zoller, Spin polarization of electrons in two-photon resonant three-photon ionization, Phys. Rev. A 24, 318 (1981).

[22] S. N. Dixit, ac-Stark-shift-induced photoelectron spin polarization in resonant multiphoton ionization, Phys. Rev. A 26, 1505 (1982).

[23] T. Herath, L. Yan, S. K. Lee, and W. Li, Strong-Field Ionization Rate Depends on the Sign of the Magnetic Quantum Number, Phys. Rev. Lett. 109, 043004 (2012).

[24] M. Ilchen, N. Douguet, T. Mazza, A. J. Rafipoor, C. Callegari, P. Finetti, O. Plekan, K. C. Prince, A. Demidovich, C. Grazioli, L. Avaldi, P. Bolognesi, M. Coreno, M. DiFraia, M. Devetta, Y. Ovcharenko, S. Düsterer, K. Ueda, K. Bartschat, A. N. Grum-Grzhimailo, A. V. Bozhevolnov, A. K. Kazansky, N. M. Kabachnik, and M. Meyer, Circular Dichroism in Multiphoton Ionization of Resonantly Excited $\mathrm{He}^{+}$Ions, Phys. Rev. Lett. 118, 013002 (2017).

[25] S. Eckart, M. Kunitski, M. Richter, A. Hartung, J. Rist, F. Trinter, K. Fehre, N. Schlott, K. Henrichs, L. P. H. Schmidt, T. Jahnke, M. Schöffler, K. Liu, I. Barth, J. Kaushal, F. Morales, M. Ivanov, O. Smirnova, and R. Dörner, Ultrafast preparation and detection of ring currents in single atoms, Nat. Phys. 14, 701 (2018).

[26] S. Eckart, K. Fehre, N. Eicke, A. Hartung, J. Rist, D. Trabert, N. Strenger, A. Pier, L. P. H. Schmidt, T. Jahnke, M. S. Schöffler, M. Lein, M. Kunitski, and R. Dörner, Direct Experimental Access to the Nonadiabatic Initial Momentum Offset upon Tunnel Ionization, Phys. Rev. Lett. 121, 163202 (2018).

[27] D. Trabert, A. Hartung, S. Eckart, F. Trinter, A. Kalinin, M. Schöffler, L. P. H. Schmidt, T. Jahnke, M. Kunitski, and R. Dörner, Spin and Angular Momentum in Strong-Field Ionization, Phys. Rev. Lett. 120, 043202 (2018).

[28] M.-M. Liu, Y. Shao, M. Han, P. Ge, Y. Deng, C. Wu, Q. Gong, and Y. Liu, Energy- and Momentum-Resolved Photoelectron Spin Polarization in Multiphoton Ionization of Xe by Circularly Polarized Fields, Phys. Rev. Lett. 120, 043201 (2018).

[29] S. Xu, Q. Zhang, X. Fu, X. Huang, X. Han, M. Li, W. Cao, and $\mathrm{P}$. Lu, Towards atom-scale spin-selective electron emitters based on strong-field Freeman resonant ionization, Phys. Rev. A 102, 063128 (2020).

[30] A. H. N. C. De Silva, D. Atri-Schuller, S. Dubey, B. P. Acharya, K. L. Romans, K. Foster, O. Russ, K. Compton, C. Rischbieter, N. Douguet, K. Bartschat, and D. Fischer, Using Circular Dichroism to Control Energy Transfer in Multiphoton Ionization, Phys. Rev. Lett. 126, 023201 (2021).
[31] I. Barth and O. Smirnova, Nonadiabatic tunneling in circularly polarized laser fields. II. Derivation of formulas, Phys. Rev. A 87, 013433 (2013).

[32] I. A. Ivanov and A. S. Kheifets, Time delay in atomic photoionization with circularly polarized light, Phys. Rev. A 87, 033407 (2013).

[33] I. Barth and O. Smirnova, Comparison of theory and experiment for nonadiabatic tunneling in circularly polarized fields, Phys. Rev. A 87, 065401 (2013).

[34] I. Barth and O. Smirnova, Spin-polarized electrons produced by strong-field ionization, Phys. Rev. A 88, 013401 (2013).

[35] J. Kaushal and O. Smirnova, Nonadiabatic Coulomb effects in strong-field ionization in circularly polarized laser fields, Phys. Rev. A 88, 013421 (2013).

[36] I. Barth and O. Smirnova, Hole dynamics and spin currents after ionization in strong circularly polarized laser fields, J. Phys. B: At., Mol. Opt. Phys. 47, 204020 (2014).

[37] I. Barth and M. Lein, Numerical verification of the theory of nonadiabatic tunnel ionization in strong circularly polarized laser fields, J. Phys. B: At., Mol. Opt. Phys. 47, 204016 (2014).

[38] F. Mauger and A. D. Bandrauk, Electronic dynamics and frequency effects in circularly polarized strong-field physics, J. Phys. B: At., Mol. Opt. Phys. 47, 191001 (2014).

[39] J. H. Bauer, F. Mota-Furtado, P. F. O’Mahony, B. Piraux, and K. Warda, Ionization and excitation of the excited hydrogen atom in strong circularly polarized laser fields, Phys. Rev. A 90, 063402 (2014)

[40] T. Wang, X.-L. Ge, J. Guo, and X.-S. Liu, Sensitivity of strongfield double ionization to the initial ensembles in circularly polarized laser fields, Phys. Rev. A 90, 033420 (2014).

[41] J. Kaushal, F. Morales, L. Torlina, M. Ivanov, and O. Smirnova, Spin-orbit Larmor clock for ionization times in one-photon and strong-field regimes, J. Phys. B: At., Mol. Opt. Phys. 48, 234002 (2015).

[42] Y. Li, P. Lan, H. Xie, M. He, X. Zhu, Q. Zhang, and P. Lu, Nonadiabatic tunnel ionization in strong circularly polarized laser fields: counterintuitive angular shifts in the photoelectron momentum distribution, Opt. Express 23, 28801 (2015).

[43] J. Kaushal, F. Morales, and O. Smirnova, Opportunities for detecting ring currents using an attoclock setup, Phys. Rev. A 92, 063405 (2015).

[44] M. H. Yuan, G. J. Zhao, and H. P. Liu, Influence of wavelength on nonadiabatic effects in circularly polarized strong-field ionization, Phys. Rev. A 92, 053405 (2015).

[45] X. Zhu, P. Lan, K. Liu, Y. Li, X. Liu, Q. Zhang, I. Barth, and P. Lu, Helicity sensitive enhancement of strong-field ionization in circularly polarized laser fields, Opt. Express 24, 4196 (2016).

[46] K. Liu and I. Barth, Nonadiabatic tunnel ionization of currentcarrying orbitals of prealigned linear molecules in strong circularly polarized laser fields, Phys. Rev. A 94, 043402 (2016).

[47] D. B. Milošević, Possibility of introducing spin into attoscience with spin-polarized electrons produced by a bichromatic circularly polarized laser field, Phys. Rev. A 93, 051402(R) (2016).

[48] J. Wätzel and J. Berakdar, Discerning on a sub-opticalwavelength the attosecond time delays in electron emission from magnetic sublevels by optical vortices, Phys. Rev. A 94, 033414 (2016). 
[49] K. Liu and I. Barth, Spin-polarized photoelectrons produced by strong-field ionization of randomly aligned nitric oxide, J. Mod. Opt. 64, 987 (2017).

[50] K. Liu, K. Renziehausen, and I. Barth, Producing spin-polarized photoelectrons by using the momentum gate in strong-field ionization experiments, Phys. Rev. A 95, 063410 (2017).

[51] J.-P. Wang and F. He, Tunneling ionization of neon atoms carrying different orbital angular momenta in strong laser fields, Phys. Rev. A 95, 043420 (2017).

[52] D. Ayuso, A. Jiménez-Galán, F. Morales, M. Ivanov, and O. Smirnova, Attosecond control of spin polarization in electronion recollision driven by intense tailored fields, New J. Phys. 19, 073007 (2017).

[53] M.-M. Liu, M. Li, Y. Shao, M. Han, Q. Gong, and Y. Liu, Effects of orbital and Coulomb potential in strong-field nonadiabatic tunneling ionization of atoms, Phys. Rev. A 96, 043410 (2017).

[54] Q. Zhang, G. Basnayake, A. Winney, Y. F. Lin, D. Debrah, S. K. Lee, and W. Li, Orbital-resolved nonadiabatic tunneling ionization, Phys. Rev. A 96, 023422 (2017).

[55] J. Kaushal and O. Smirnova, Looking inside the tunnelling barrier: I. Strong field ionisation from orbitals with high angular momentum in circularly polarised fields, J. Phys. B: At., Mol. Opt. Phys. 51, 174001 (2018).

[56] J. Kaushal and O. Smirnova, Looking inside the tunnelling barrier: II. Co- and counter-rotating electrons at the 'tunnelling exit', J. Phys. B: At., Mol. Opt. Phys. 51, 174002 (2018).

[57] J. Kaushal and O. Smirnova, Looking inside the tunnelling barrier III: spin polarisation in strong field ionisation from orbitals with high angular momentum, J. Phys. B: At., Mol. Opt. Phys. 51, 174003 (2018).

[58] D. D. A. Clarke, G. S. J. Armstrong, A. C. Brown, and H. W. van der Hart, $R$-matrix-with-time-dependence theory for ultrafast atomic processes in arbitrary light fields, Phys. Rev. A 98, 053442 (2018).

[59] K. Liu, H. Ni, K. Renziehausen, J.-M. Rost, and I. Barth, Deformation of Atomic $p_{ \pm}$Orbitals in Strong Elliptically Polarized Laser Fields: Ionization Time Drifts and Spatial Photoelectron Separation, Phys. Rev. Lett. 121, 203201 (2018).

[60] S.-D. Jheng, T.-F. Jiang, J.-H. Chen, and J.-L. Liu, Magnetic quantum number dependence of hydrogen photoelectron spectra under circularly polarized pulse in barrier suppression ionization regime, Phys. Scr. 93, 085401 (2018).

[61] M. Han, P. Ge, M.-M. Liu, Q. Gong, and Y. Liu, Spatially and temporally controlling electron spin polarization in strong-field ionization using orthogonal two-color laser fields, Phys. Rev. A 99, 023404 (2019).

[62] G. S. J. Armstrong, D. D. A. Clarke, A. C. Brown, and H. W. van der Hart, Electron rotational asymmetry in strong-field photodetachment from $\mathrm{F}^{-}$by circularly polarized laser pulses, Phys. Rev. A 99, 023429 (2019).

[63] J. Hofbrucker, A. V. Volotka, and S. Fritzsche, Fluorescence polarization as a precise tool for understanding nonsequential many-photon ionization, Phys. Rev. A 100, 011401(R) (2019).

[64] D. Wu, F.-M. Guo, J.-G. Chen, J. Wang, and Y.-J. Yang, Ionization of an atom with different initial angular momenta in an intense circular polarized laser field, J. Phys. B: At., Mol. Opt. Phys. 53, 235601 (2020).

[65] J. H. Bauer and Z. Walczak, Ionization and excitation of lowlying circular states of the hydrogen atom in strong circularly polarized laser fields, Phys. Rev. A 101, 063409 (2020).

[66] G. S. J. Armstrong, D. D. A. Clarke, J. Benda, A. C. Brown, and H. W. van der Hart, Electron correlation and short-range dynamics in attosecond angular streaking, Phys. Rev. A 101, 041401(R) (2020).

[67] E. V. Gryzlova, M. M. Popova, and A. N. Grum-Grzhimailo, Spin polarization of photoelectrons in bichromatic extremeultraviolet atomic ionization, Phys. Rev. A 102, 053116 (2020).

[68] B. Böning, P. Abele, W. Paufler, and S. Fritzsche, A strong-field approach with realistic wave functions to the above-threshold ionization of $\mathrm{Ba}^{+}$, J. Phys. B: At., Mol. Opt. Phys. 54, 025602 (2021).

[69] A. H. N. C. De Silva, T. Moon, K. L. Romans, B. P. Acharya, S. Dubey, K. Foster, O. Russ, C. Rischbieter, N. Douguet, K. Bartschat, and D. Fischer, Circular dichroism in atomic resonance-enhanced few-photon ionization, Phys. Rev. A 103, 053125 (2021).

[70] S. Walker, L. Kolanz, J. Venzke, and A. Becker, Enhanced ionization of counter-rotating electrons via doorway states in ultrashort circularly polarized laser pulses, Phys. Rev. A 103, L061101 (2021).

[71] G. S. J. Armstrong, D. D. A. Clarke, J. Benda, J. Wragg, A. C. Brown, and H. W. van der Hart, Enhancing spin polarization using ultrafast angular streaking, Phys. Rev. A 103, 053123 (2021).

[72] R. R. Freeman, P. H. Bucksbaum, H. Milchberg, S. Darack, D. Schumacher, and M. E. Geusic, Above-Threshold Ionization with Subpicosecond Laser Pulses, Phys. Rev. Lett. 59, 1092 (1987).

[73] R. Reiff, T. Joyce, A. Jaroń-Becker, and A. Becker, Singleactive electron calculations of high-order harmonic generation from valence shells in atoms for quantitative comparison with TDDFT calculations, J. Phys. Commun. 4, 065011 (2020).

[74] We note that in our previous work [70] we used a wrong sign in front of the second term and did not include the factor $1 / 1+\epsilon^{2}$ for $A_{0}$ when defining the vector potential. The actual numerical calculations in [70] are however not impacted by this mistake in the text of the article.

[75] J. Venzke, T. Joyce, Z. Xue, A. Becker, and A. Jaron-Becker, Central frequency of few-cycle laser pulses in strong-field processes, Phys. Rev. A 98, 063409 (2018).

[76] J. Venzke, A. Jaron-Becker, and A. Becker, Ionization of helium by an ultrashort extreme-ultraviolet laser pulse, J. Phys. B: At., Mol. Opt. Phys. 53, 085602 (2020).

[77] S. Chen, X. Gao, J. Li, A. Becker, and A. Jaroń-Becker, Application of a numerical-basis-state method to strong-field excitation and ionization of hydrogen atoms, Phys. Rev. A 86, 013410 (2012).

[78] S. Walker, R. Reiff, A. Jaron-Becker, and A. Becker, Characterization of vacuum and deep ultraviolet pulses via two-photon autocorrelation signals, Opt. Lett. 46, 3083 (2021).

[79] B. Brandsen and C. Joachain, Physics of Atoms and Molecules (Addison-Wesley, London, 2003). 